\title{
Modèle numérique pour l'étude des structures fissurées soumises à des contraintes résiduelles
}

\author{
Zohra Gaiech* — Hocine Kebir* _ Laurent Chambon** \\ Jean-Marc Roelandt* \\ * Laboratoire Roberval - UTC-CNRS, Université de Technologie de Compiègne \\ GSM - Centre de Recherches de Royalieu \\ BP 20529, F-60200 Compiègne \\ zohra_gaiech@yahoo.fr \\ hocine.kebir@utc.fr \\ ** EADS, Centre Commun de Recherches \\ 12, Rue Pasteur BP 76, F-92152 Suresnes cedex
}

\begin{abstract}
RÉSUMÉ. Au cours de leur utilisation opérationnelle, les structures aéronautiques peuvent être soumises à des charges cycliques relativement modérées et plus rarement à des charges sévères. Ces dernières peuvent cependant générer des contraintes résiduelles, qui vont influer sur le comportement en service. Par ailleurs, des contraintes peuvent être créées d'une façon volontaire par un traitement spécifique (contraintes de compression). Elles permettent d'améliorer la résistance en fatigue. Ceci illustre l'importance de l'étude du comportement des structures en présence de ces contraintes internes, et le besoin de les considérer lors de simulations numériques utilisées. L'objectif de nos travaux est de développer un modèle numérique basé sur la méthode des équations intégrales duales et la méthode de superposition, qui permet de mettre en évidence l'effet des contraintes résiduelles sur la tolérance au dommage des structures métalliques.

ABSTRACT. During their operational use the aeronautic structures can be submitted to relatively moderate cyclic loads and more rarely to stern loads. These last can generate residual stress field, which will influence the in-service behaviour. Otherwise, residual stress field can be created in a voluntary way by a specific treatment (compressive stresses). They permit to improve fatigue tolerance behaviour. This illustrates the importance of the study of the structure behavior under residual stresses, and the need to consider them in numerical simulations. The objective of this work is to develop a numerical method, based on the boundary element method and the principle of superposition, to assess the influence of residual stresses on fracture mechanics parameters.
\end{abstract}

MOTS-CLÉS: contraintes résiduelles, mécanique de la rupture, équations intégrales, méthode de superposition.

KEYWORDS: residual stresses, fracture mechanics, boundary element, superposition principle.

DOI:10.3166/REMN.16.627-642 (C) Lavoisier, Paris 


\section{Introduction}

La méthode des équations intégrales s'avère très efficace pour traiter les problèmes de propagation de fissures en mécanique linéaire de la rupture.

Les bases de cette méthode ont été jetées par Fredholm et Mikhilin, grâce à une formulation mathématique classique basée sur la théorie des potentiels. Betti, Somigliana et Kupradze l'ont étendue à l'élasticité. Le travail de Lachat (1975) et Lachat et Watson (1976) est probablement le travail le plus significatif qui a permis de faire de la méthode des éléments finis de frontières une technique numérique effective, par discrétisation des équations intégrales. Ils ont développé une formulation iso-paramétrique similaire à la méthode des éléments finis et ils ont montré que cette méthode peut être utilisée comme un outil efficace pour résoudre des problèmes à configurations complexes. Les travaux réalisés au sein du laboratoire Roberval (Kebir et al., 1999), ont conduit au développement d'un code Prf2d, intégré par EADS CCR comme solveur dans le logiciel CRACK- KIT. Ce logiciel permet de simuler le comportement des structures bidimensionnelles fissurées ou non fissurées.

Le modèle numérique développé utilise des éléments de frontière quadratiques non conformes et des éléments singuliers en fonds de fissure développés à partir de la méthode des équations intégrales duales (Brebbia et al., 1989; Portela et al., 1992 ; Bonnet 1994). Ce modèle est associé à des procédures numériques de propagation de fissures. La prise en compte des contraintes résiduelles dans les calculs de mécanique de la rupture nécessite a priori, quelle que soit la méthode numérique utilisée, un calcul non linéaire. Cependant, lorsque les déformations résiduelles sont faibles et leur redistribution au cours de la fissuration peu importantes, on peut considérer en première approximation que le champ de contrainte résiduel détermine seul le comportement en fissuration. Moyennant cette approximation, on peut faire abstraction des déformations et utiliser la superposition du champ de contrainte résiduel et du champ (linéaire) dû à la sollicitation pour calculer les paramètres usuels de mécanique de la rupture. L'effet des contraintes résiduelles est introduit dans nos calculs à l'aide de cette méthode de superposition.

Dans la suite, on présente tout d'abord, les équations intégrales duales nécessaires pour traiter le problème de propagation de fissure en présence des contraintes résiduelles en élasticité. On décrit dans les sections suivantes le traitement numérique de ces équations intégrales ainsi que les procédures numériques associées à la propagation de fissures.

Enfin, on présente une comparaison entre les résultats de calcul et expérimentaux sur des tests de ténacité d'éprouvette entaillée préalablement comprimée. 


\section{Formulation des équations intégrales}

La formulation est présentée dans le cadre de l'étude d'une structure bidimensionnelle occupant un domaine $\Omega$ et de frontière $\partial \Omega$. La combinaison de l'équation d'équilibre d'un solide élastique linéaire, du théorème de réciprocité de Maxwell-Betti et de la solution élémentaire du problème de Kelvin nous permet d'obtenir l'équation [1] qui permet de résoudre en général un problème élastostatique en présence de contraintes initiales $\sigma^{I}$.

$C_{l k}^{P} u_{k}^{P}(P)+\int_{\partial \Omega} T_{l k}(P, M) u_{k}(M) d S=\int_{\partial \Omega} U_{l k}(P, M) t_{k}(M) d S-\int_{\Omega} \sigma_{j k}^{I}(M) E_{l j k}(P, M) d V$

avec $C_{l k}^{P}= \begin{cases}\frac{1}{2} \delta_{l k} & \text { si } P \in \partial \Omega \\ 1 & \text { si } P \in \Omega \\ 0 & \text { si } P \text { est à l'extérieur }\end{cases}$

$U_{l k}$ et $T_{l k}$ sont les solutions fondamentales du problème de Kelvin obtenues de l'équation de Navier pour une charge ponctuelle unitaire appliquée au point $\mathrm{P}$ dans une direction $l$ :

$U_{l k}$ est le déplacement en un point $\mathrm{M}$ du domaine $\Omega$ dans la direction $k$ où la charge unitaire est appliquée au point $\mathrm{P}$ dans la direction $l$.

$T_{l k}$ est le vecteur tension au point M sur une facette de normale $\vec{n}$.

$$
\begin{aligned}
& U_{l k}(P, M)=\frac{1}{8 \pi \mu(1-v)}\left[(3-4 v) L n\left(\frac{1}{r}\right) \delta_{l k}+r_{, l} r_{, k}\right] \\
& T_{l k}(P, M)=\frac{-1}{4 \pi(1-v) r}\left[\frac{\partial r}{\partial n}\left((1-2 v) \delta_{l k}+2 r_{, k} r_{, l}\right)+(1-2 v)\left(n_{l} r_{, k}-n_{k} r_{l}\right)\right]
\end{aligned}
$$

avec :

$v$ : coefficient de poisson, $\mu$ : coefficient de cisaillement

$r$ est la distance entre $\mathrm{P}$ et $\mathrm{M}$ 


$$
\vec{r}=\overrightarrow{P M} \quad r_{, l}=\frac{\partial r}{\partial x_{l}}=\frac{r_{l}}{r} \quad r_{, k}=\frac{\partial r}{\partial x_{k}}=\frac{r_{k}}{r}
$$

$r_{l}$ et $r_{k}$ sont les projections respectives de $r$ dans la direction $l$ et $k$.

$$
\frac{\partial r}{\partial n}=r_{, i} n_{i}=r_{, 1} n_{1}+r_{, 2} n_{2}
$$

et $\delta_{i j}$ est l'indice de Kronecker défini par: $\delta_{i j}=\left\{\begin{array}{ccc}1 & \text { si } & i=j \\ 0 & \text { si } & i \neq j\end{array}\right.$

$E_{l j k}$ définit le tenseur des déformations fondamentales obtenu à partir de $U_{l k}$ :

$$
E_{l j k}(P, M)=\frac{1}{8 \pi \mu(1-v) r}\left[(2 v-1)\left(r_{, k} \delta_{l j}+r_{, j} \delta_{l k}\right)+\delta_{j k, l} r_{,}-2 r_{, l} r_{, k} r_{, j}\right]
$$

La méthode des équations intégrales duales consiste à écrire l'équation intégrale en déplacement sur les points d'une lèvre de la fissure et à écrire son équation duale (l'équation intégrale en tension) sur l'autre lèvre. Dans ce qui suit, on présente les équations intégrales nécessaires pour traiter le problème de structure fissurée en présence des contraintes résiduelles.

\subsection{Equation intégrale en déplacement pour les points du contour}
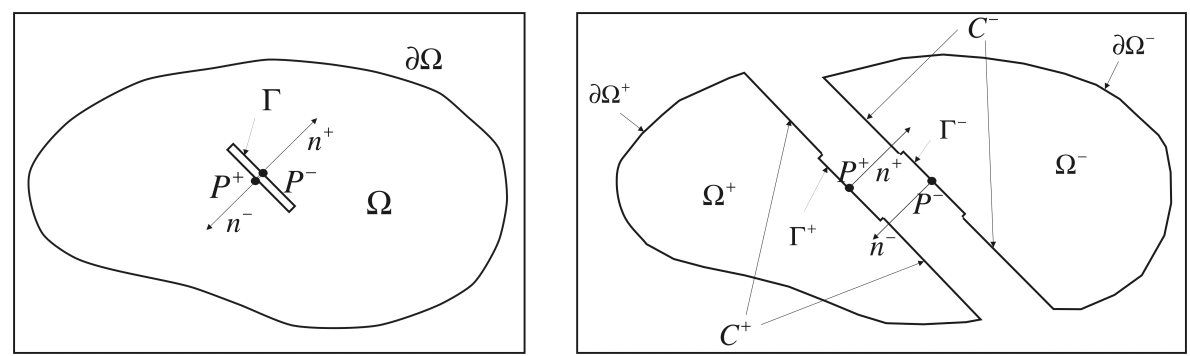

Figure 1. Structure fissurée 
La structure fissurée est décomposée par la pensée en deux domaines $\Omega^{+}$et $\Omega$ séparés par un contour $\mathrm{C}$ et la fissure $\Gamma$. L'équation intégrale en déplacement pour $\mathrm{P}$ appartenant à $\Omega$ s'écrit :

$$
\underline{1}_{2} u_{k}(P)+\int_{\partial \Omega \cup \Gamma} T_{l k}(P, M) u_{k}(M) d S=\int_{\partial \Omega \cup \Gamma} U_{l k}(P, M) t_{k}(M) d S-\int_{\Omega} E_{l m k}(P, M) \sigma_{m k}^{I}(M) d V
$$

\subsection{Equation intégrale en déplacement pour les points de la fissure}

L'équation intégrale en déplacement pour un point $\mathrm{P}$ situé sur les lèvres de la fissure est donnée par l'équation [7] ci-après.

$$
\frac{1}{2}\left(u_{k}\left(P^{+}\right)+u_{k}\left(P^{-}\right)\right)+\int_{\partial \Omega \cup \Gamma} T_{l k}(P, M) u_{k}(M) d S=\int_{\partial \Omega \cup \Gamma} U_{l k}(P, M) t_{k}(M) d S-\int_{\Omega} E_{l m k}(P, M) \sigma_{m k}^{I}(M) d V \text { [7] }
$$

Cette équation est obtenue par la sommation de deux équations en déplacements écrites au point $\mathrm{P}=\mathrm{P}^{+}$situé sur la lèvre $\Gamma^{+}$de la fissure et au point $\mathrm{P}=\mathrm{P}^{-}$situé sur la lèvre $\Gamma^{-}$de la fissure, tenant compte des conditions de raccord et d'opposition de normales sur les lèvres.

\subsection{Equation intégrale en tension pour les points de la fissure}

L'équation intégrale en contrainte pour un point $\mathrm{P}=\mathrm{P}^{+}=\mathrm{P}^{-}$situé sur les lèvres de la fissure s'écrit :

$$
\begin{aligned}
\frac{1}{2}\left(\sigma_{i j}\left(P^{+}\right)+\sigma_{i j}\left(P^{-}\right)\right)= & \int_{\partial \Omega \cup \Gamma} D_{i j k}(P, M) t_{k}(M) d S-\int_{\partial \Omega \cup \Gamma} S_{i j k}(P, M) u_{k}(M) d S \\
& -\int_{\Omega} F_{i j m k}(P, M) \sigma_{m k}^{I}(M) d \Omega+2 g_{i j}\left(\sigma_{m k}^{I}\right)
\end{aligned}
$$

$D_{i j k}, S_{i j k}$ (Lachat, 1975 ; Kebir et al., 2000 ; Aliabadi, 2002) et $F_{i j m k}$ sont des termes obtenus à partir de la loi de Hooke et la solution élémentaire de Kelvin. On ne présente que les termes associés aux contraintes initiales.

$$
\begin{gathered}
F_{i j m k}=\frac{1}{4 \pi(1-v) r^{2}}\left[( 2 v - 1 ) \left(\delta_{k j} \delta_{i m}+\delta_{m j} \delta_{i k}-\delta_{m k} \delta_{i j}-r_{, k} r_{, j} \delta_{i m}-r_{, k} r_{, i} \delta_{j m}-r_{, m} r_{, j} \delta_{i k}-r_{, m} r_{, i} \delta_{j k}\right.\right. \\
\left.\left.+2 r_{, k} r_{, m} \delta_{i j}\right)-2 r_{, i} r_{j} \delta_{m k}-r_{, i} r_{m} \delta_{k j}-r_{, i, k} r_{m j} \delta_{, j, m}-r_{k i} \delta_{k i, j}-r_{, k} \delta_{m i}+8 r_{, i, j} r_{, m} r_{, k}\right] \\
g_{i j}=-\frac{1}{8(1-v)}\left[2 \sigma_{i j}^{I}+(1-4 v) \sigma_{l l}^{I} \delta_{i j}\right]
\end{gathered}
$$


La multiplication des deux membres de l'équation [8] par la normale $\mathrm{n}^{+}$du point $\mathrm{P}^{+}$ou $\mathrm{n}^{-}$du point $\mathrm{P}^{-}$nous donne l'équation intégrale en tension :

$$
\begin{aligned}
\underline{1}_{2}\left(t_{i}\left(P^{+}\right)+t_{i}\left(P^{-}\right)\right) & =n_{j}\left(P^{+}\right) \int_{\partial \Omega \cup \Gamma} D_{i j k}(P, M) t_{k}(M) d S-n_{j}\left(P^{+}\right) \int_{\partial \Omega \cup \Gamma} S_{i j k}(P, M) u_{k}(M) d S \\
& -n_{j}\left(P^{+}\right) \int_{\Omega} F_{i j m k}(P, M) \sigma_{m k}^{I}(M) d \Omega+2 n_{j}\left(P^{+}\right) g_{i j}\left(\sigma_{m k}^{I}\right)
\end{aligned}
$$

\section{Traitement numérique des équations intégrales duales}

\subsection{Discrétisation de la géométrie et des champs élastiques}

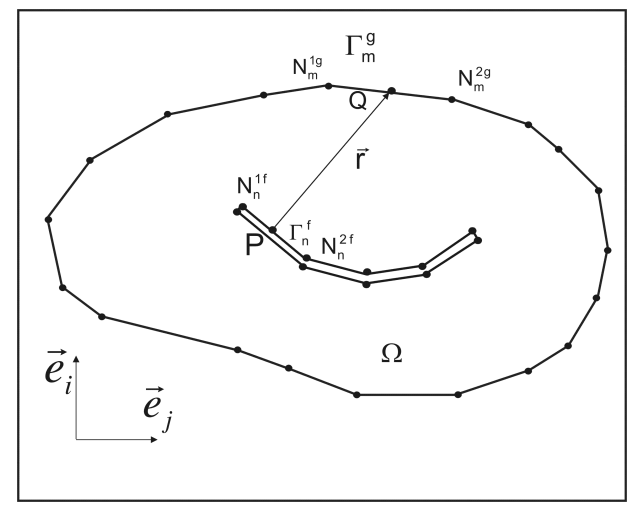

Figure 2. Discrétisation de la géométrie en éléments de frontières

Le contour $\partial \Omega$ de la structure est discrétisé en NEG segments de droite $\Gamma_{m}^{g}(\mathrm{~m}=1, \mathrm{NEG})$. La fissure est discrétisée en $2 \times \mathrm{NEF}$ segments de droite $\Gamma_{m}^{f}(\mathrm{~m}=1$, $2 \times \mathrm{NEF}$ ). Par contre le domaine $\Omega$ est subdivisé en $\mathrm{M}$ éléments internes sur lesquels les intégrales de volume sont évaluées numériquement.

Les champs élastiques en déplacement et de tension $(\vec{u}(Q), \vec{t}(Q), \mathrm{Q} \in \partial \Omega \cup \Gamma)$ sont interpolés par des fonctions de forme quadratiques. Les trois points de collocation ont été choisis à l'intérieur de l'élément de discrétisation comme le montre la figure 3. Le fond de la fissure est discrétisé à l'aide d'éléments singuliers (Kebir et al., 1999) permettant de représenter exactement le champ des déplacements. Cet élément singulier contient trois points de collocation positionnés de la même façon que ceux de l'élément quadratique non conforme mais les fonctions de forme sont comme suit : 
$N_{i}(s)=a_{i}+b_{i} s+c_{i} \sqrt{s}$

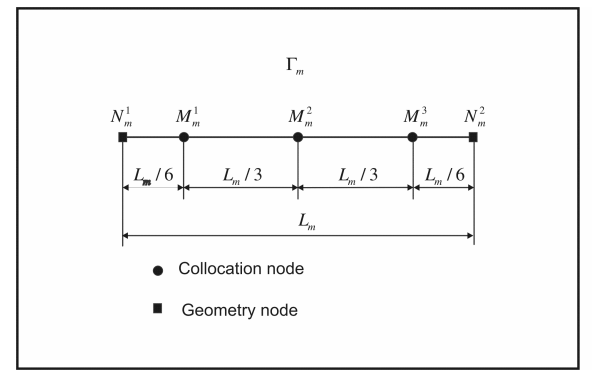

Elément de géométrie quadratique

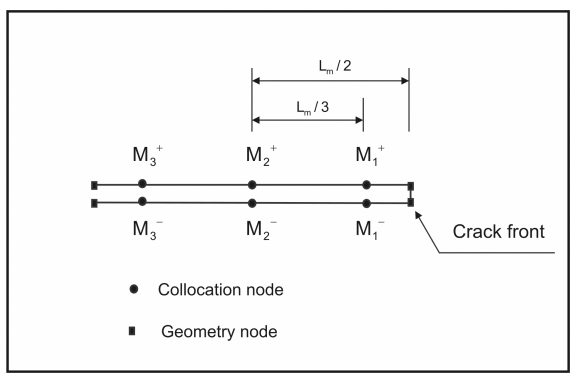

Elément de fond de fissure

Figure 3. Eléments de discrétisation de la géométrie

\subsection{Traitement des intégrales}

Les intégrales de volume présentent deux types de singularité, une singularité faible en $(1 / \mathrm{r})$ et une singularité forte en $\left(1 / \mathrm{r}^{2}\right)$. L'évaluation de ces intégrales nécessite une subdivision du domaine $\Omega$ en cellules d'intégration. Si le point de collocation appartient à l'élément considéré, on utilise une intégration singulière, sinon on intègre de façon régulière (Lachat et Watson, 1976 ; Leitao et Aliabadi, 1995). Ces intégrales sont donc évaluées au sens de la valeur principale de Cauchy de façon analytique sur les éléments singuliers. Les intégrales de surface singulières sont également évaluées de façon analytique (Kebir et al., 2000).

\section{Calcul des structures fissurées}

\subsection{Principe de la méthode de superposition}

Comme son nom l'indique, la méthode de superposition consiste à décomposer un problème complexe en des sous-problèmes élémentaires, dont la résolution est plus facile. Elle est applicable en élasticité linéaire et petites perturbations, grâce à la linéarité des équations.

Dans notre cas, notre problème $(\mathrm{P})$ est décomposé en deux sous-problèmes $(\mathrm{P} 1)$ et (P2), (voir figure 4).

(P) est le problème qu'on veut résoudre.

(P1) : on considère seulement les contraintes initiales dans le domaine et les efforts de compression appliqués sur les lèvres de la fissure évalués à partir de l'état initial. 
(P2) : on considère les efforts appliqués sur la frontière et les efforts de traction appliqués sur les lèvres de la fissure opposés à ceux du problème $(\mathrm{P} 1)$.

Dans l'étude présentée, ces problèmes sont résolus avec l'hypothèse de contrainte plane uniquement.
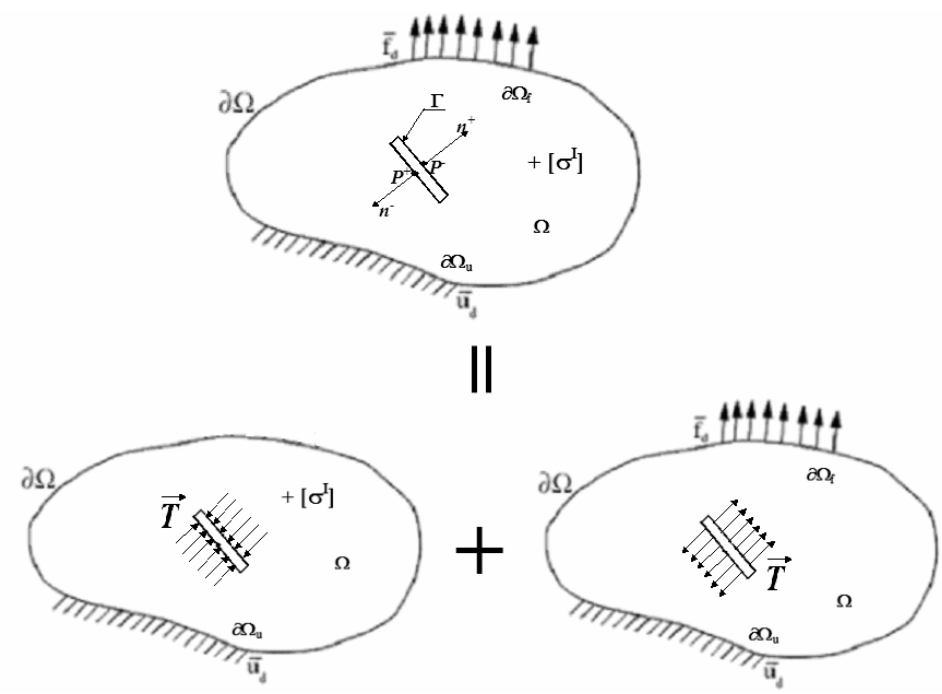

Figure 4. Principe de la méthode de superposition

\subsection{Calcul des facteurs d'intensité de contraintes}

Le code Prf2d, mis en œuvre dans cette étude, utilise la méthode d'extrapolation des déplacements au voisinage du fond de la fissure pour évaluer les facteurs d'intensité de contraintes $K_{I}$ et $K_{I I}$.

En considérant l'élément de fond de fissure (figure 3 ) et après résolution du problème (déjà posé), une extrapolation linéaire des valeurs des facteurs d'intensité de contraintes entre les points $\mathrm{M}_{3}$ et $\mathrm{M}_{2}$ vers le fond de la fissure nous permet d'avoir le système d'équation ci-après.

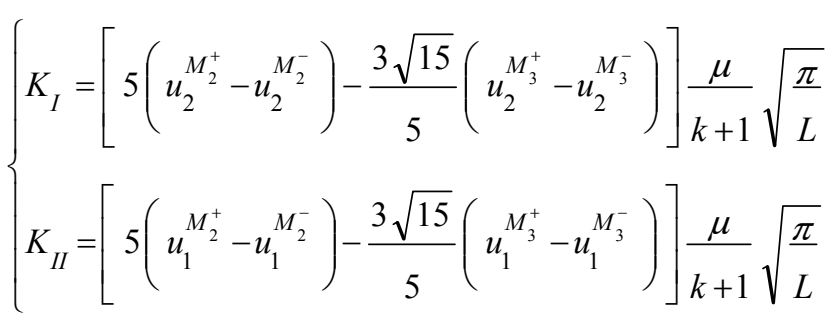




\section{Validation numérique}

\subsection{Préambule}

Certaines zones fortement sollicitées sur des structures aéronautiques peuvent présenter des déformations plastiques localisées sous charge limite, qui induisent des contraintes résiduelles après retour élastique. Des essais reproduisant ce type de phénomène ont été menés sur des éprouvettes entaillées, afin d'évaluer l'influence des contraintes résiduelles sur leur ténacité. L'essai est mené selon les principes de l'ASTME1820 à EADS-CCR (en particulier pour la préfissuration).

La géométrie de l'éprouvette est présentée sur la figure 5. L'éprouvette est prélevée dans une plaque d'épaisseur $30 \mathrm{~mm}$ et de module de Young $\mathrm{E}=68500 \mathrm{MPa}$ et d'un coefficient de poisson $v=0.33$.

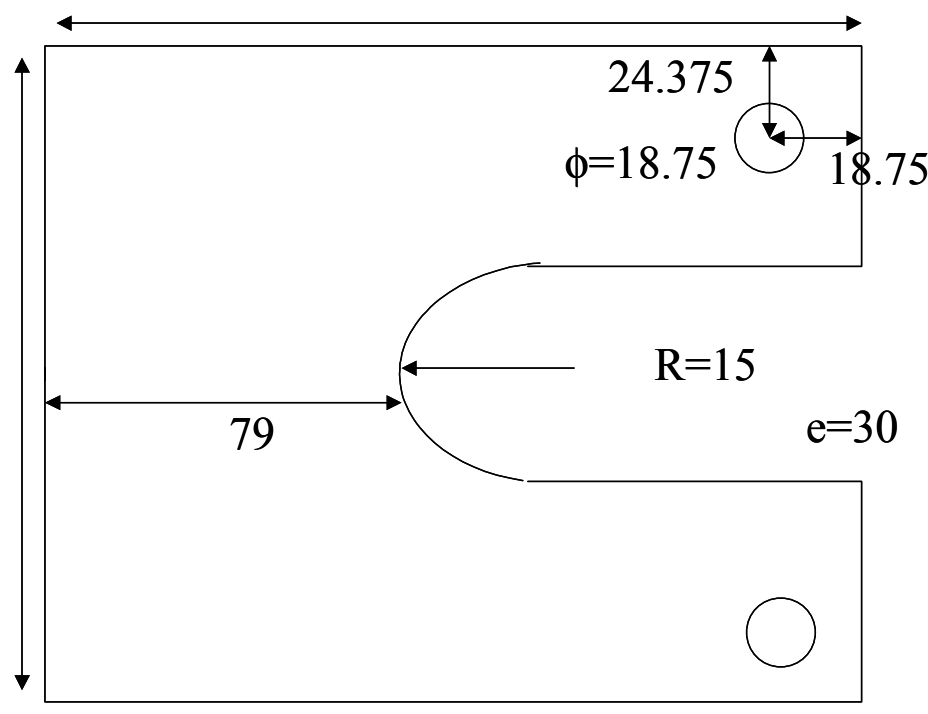

Figure 5. Géométrie de l'éprouvette

\subsection{Etat de contraintes résiduelles}

L'état de contraintes résiduelles est obtenu par un calcul non linéaire, sous hypothèse de contrainte plane, en appliquant une compression de $1000 \mathrm{kN}$ sur la face supérieure à l'aide d'un plateau rigide puis un retour élastique à $\mathrm{O} \mathrm{kN}$. Le contact entre le plateau et l'éprouvette est réalisé avec un coefficient de frottement $=0.3$. Ce calcul éléments finis est réalisé avec une condition de symétrie par rapport à l'axe de géométrie de l'éprouvette. La figure 6 présente la distribution des contraintes résiduelles de Von-Mises dans l'éprouvette. On remarque, que le champ de contraintes obtenu est un champ de contraintes de traction symétrique par 
rapport à l'axe de symétrie géométrique de l'éprouvette. On constate l'existence de trois zones : une première zone où les contraintes sont très élevées, au niveau de l'alésage, une zone de transition où les contraintes diminuent puis restent faibles, et une dernière zone où les contraintes sont quasiment nulles.
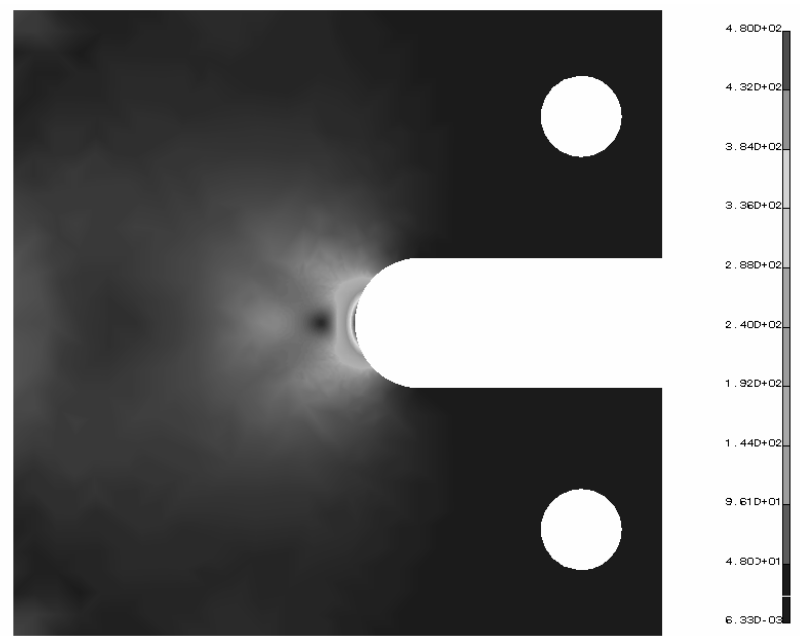

Figure 6. Distribution des contraintes initiales de Von-Mises dans l'éprouvette

\subsection{Détermination du facteur d'intensité de contraintes dû à l'état des contraintes} initial

Le modèle éléments de frontières et le modèle éléments finis, dont les mailles sont utilisées seulement comme cellules d'intégration, sont donnés par la figure 7 .

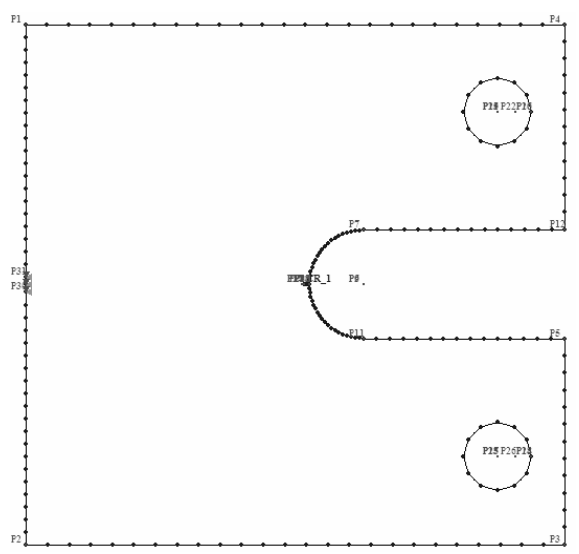




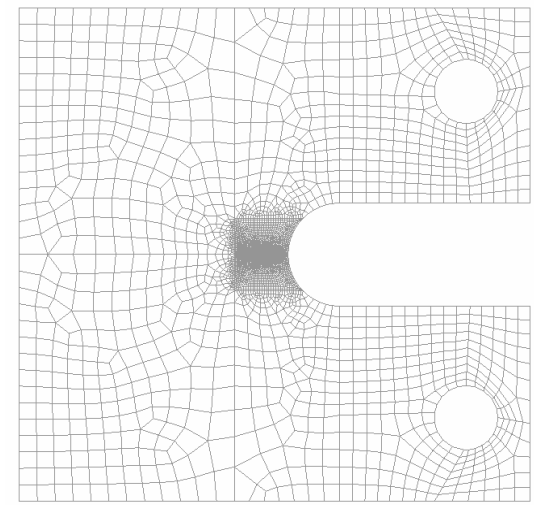

Figure 7. Modèle éléments de frontières et éléments finis

Le facteur d'intensité de contraintes est calculé pour trois types de maillages, de densité croissante sur le plan de fissure. Les tailles de maille testées au niveau de ce plan sont : 0,5 mm, $0,158 \mathrm{~mm}$ et $0,079 \mathrm{~mm}$.

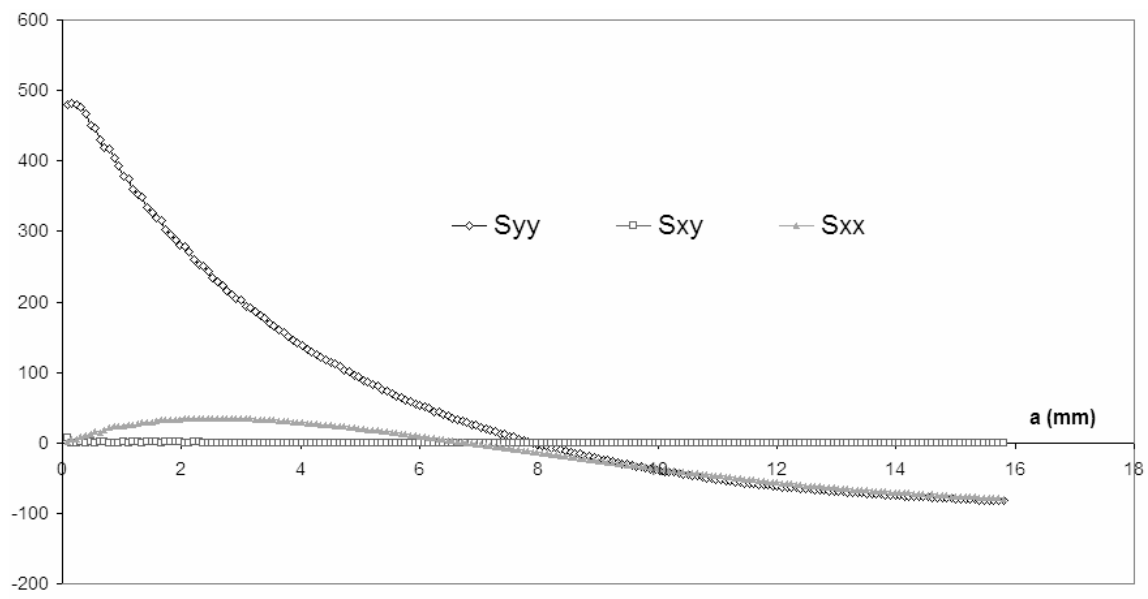

Figure 8. Distribution des contraintes résiduelles sur le plan de fissure

Sur la figure 9, on a reporté les résultats obtenus pour les trois maillages définis précédemment et sur la figure 8 la distribution des contraintes résiduelles sur le plan de fissure (maillage 2).

On remarque que pour les fissures courtes, jusqu'à une longueur de $3 \mathrm{~mm}$, le facteur KI augmente puis au-delà de cette longueur ce facteur diminue 
progressivement (figure 9). Ce comportement qualitatif est conforme à l'intuition. En effet, cela est dû à l'existence d'une zone de concentration des contraintes au voisinage de l'alésage. Le facteur d'intensité de contrainte est fonction de deux paramètres : la valeur des contraintes sur les lèvres de fissure d'une part, et la longueur des lèvres, d'autre part. Comme ces deux paramètres varient en sens opposé (voir figure 8), il est logique de trouver un résultat non monotone.

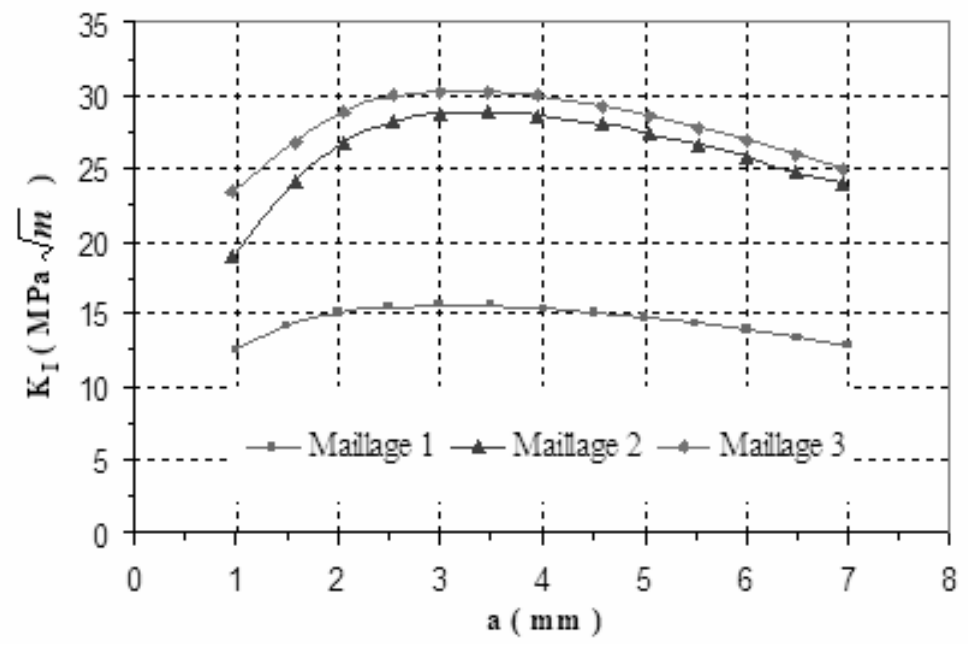

Figure 9. Résultats obtenus pour les trois types de maillage

On remarque qu'en raffinant le maillage, on constate une convergence des résultats. La taille de maille raisonnable pour considérer le résultat comme convergé est de l'ordre de $0,1 \mathrm{~mm}$. Ceci s'explique par le fait que l'estimation des contraintes est sensible à la taille de maille dans les zones de fort gradient, et que l'estimation du facteur d'intensité de contrainte par superposition est notoirement sensible à la contrainte estimée près de la pointe de la fissure. A titre d'exemple, pour le maillage le plus grossier, il n'y a qu'un seul élément dans chacune des trois zones définies ciavant, ce qui est évidemment insuffisant. Il faut raffiner plus autour de la zone de concentration des contraintes pour mieux capter l'effet des contraintes initiales sur l'ouverture de la fissure.

\subsection{Comparaison avec les résultats expérimentaux}

Afin d'avoir une comparaison exacte avec les facteurs d'intensité de contraintes obtenus expérimentalement (sur la base des essais réalisés par EADS CCR) on doit superposer les résultats obtenus avec les facteurs d'intensité de contraintes dus au chargement en traction expérimental. Pour cela on a utilisé, la charge moyenne à la 
rupture qui est de l'ordre de 94248 N. Cette charge est appliquée au niveau des deux alésages (voir figure 10) par l'intermédiaire de deux autre pièces dont le matériau est caractérisé par un module de Young $\mathrm{E}=70000 \mathrm{MPa}$ et un coefficient de poisson $v=0,3$.

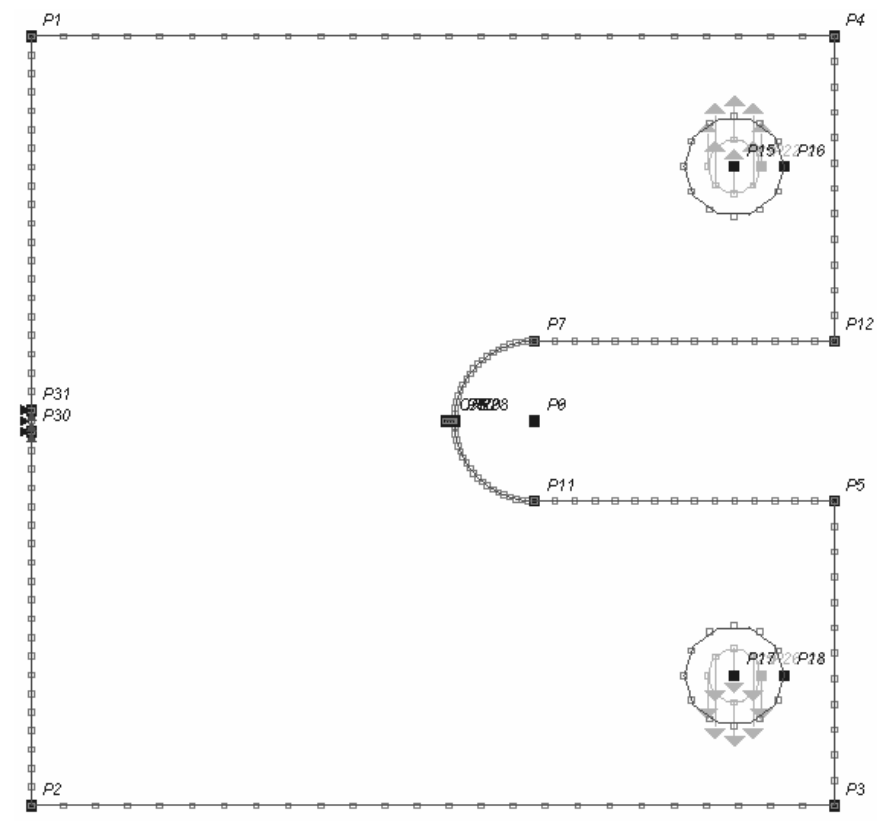

Figure 10. Plaque sous chargement

Sur la figure 11 on présente les facteurs d'intensité de contraintes (F.I.C) effectifs (obtenus par superposition des deux calculs) et les facteurs d'intensité de contraintes expérimentaux. La figure 11 montre que les facteurs d'intensité de contraintes effectifs et expérimentaux sont très proches (à $2 \mathrm{MPa} \sqrt{m}$ près). Cette corrélation doit être nuancée par deux observations. D'une part, l'estimation du facteur d'intensité de contrainte d'amorçage du défaut par la méthode de la sécante (ASTME 1820) est sensible au choix d'un coefficient dit coefficient d'abattement. Pour une éprouvette non normalisée, il n'existe pas, par définition, d'abattement standardisé. L'utilisation de divers coefficients réalistes montre que l'estimation du facteur d'intensité de contrainte à l'amorçage peut varier d'environ $10 \%$ selon l'hypothèse retenue. D'autre part, l'observation du faciès après rupture révèle une propagation en tunnel beaucoup plus importante en présence de contraintes résiduelles. En conséquence, la validité du facteur d'intensité de contrainte comme indicateur d'amorçage sur la base d'une variation de compliance est sujette à caution, puisque le front de propagation n'est pas rectiligne. En conséquence, les valeurs expérimentales doivent être considérées comme des indicateurs d'amorçage 
plutôt que comme des mesures. Dans cette optique, la méthode de calcul proposée apparaît également comme un bon estimateur de la charge critique, permettant d'appréhender au moins au premier ordre l'effet des contraintes résiduelles (à savoir la réduction d'environ $50 \%$ de la charge critique).

Ce premier calcul avec un état des contraintes résiduelles récupéré par un calcul éléments finis non linéaire valide les développements réalisés. L'extension de la méthode à des chargements de fatigue, et à des profils de contrainte complexes (dus au soudage notamment) mérite d'être envisagée.

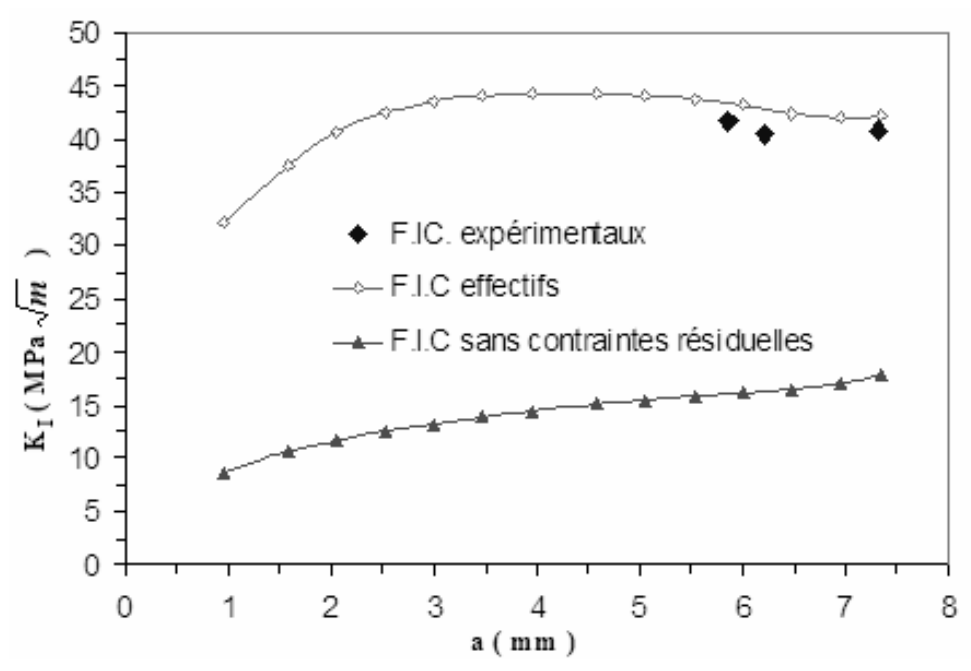

\begin{tabular}{lccc}
\hline $\mathrm{a}(\mathrm{mm})$ & $\mathrm{K}_{\mathrm{I}}$ experimental $(\mathrm{MPa} \sqrt{\boldsymbol{m}})$ & $\mathrm{K}_{\mathrm{I}}$ numérique $(\mathrm{MPa} \sqrt{\boldsymbol{m}})$ & Ecart (\%) \\
\hline 5.86 & 41.71 & 43.34 & 3.9 \\
6.225 & 40.46 & 42.839 & 5.88 \\
7.315 & 40.87 & 42.106 & 3.024 \\
\hline
\end{tabular}

Figure 11. Evolution de $K_{I}$ en fonction de la longueur de la fissure a

\section{Conclusions et perspectives}

Un modèle numérique permettant de traiter les problèmes d'ouverture de fissures en présence de contraintes initiales sous chargement dans le domaine élastique, a été mis au point. Ce modèle est basé sur la méthode de superposition et la méthode des 
équations intégrales duales. Les intégrales de surface qui apparaissent dans la formulation sont évaluées de façon numérique à l'aide de la méthode de Gauss (intégrales régulières) et analytique (intégrales singulières). La prédiction de la réduction de ténacité sur une éprouvette entaillée préalablement comprimée est conforme, au premier ordre, à la réduction constatée par essai. En effet, la prévision de la charge d'amorçage du défaut est très bonne, mais la sensibilité des valeurs expérimentales aux hypothèses de dépouillement, ainsi que l'analyse post-mortem du scénario de rupture sur les faciès incite à considérer la méthode proposée comme fournissant seulement un bon ordre de grandeur de la résistance résiduelle.

Un modèle numérique permettant de traiter les problèmes de propagation des fissures a été aussi mis au point. Mais vue que le calcul préalable du champ des contraintes résiduelles est réalisé en considérant la symétrie géométrique de l'éprouvette, les résultats obtenus avec et sans propagation des fissures sont pratiquement identiques.

\section{Bibliographie}

Aliabadi M. H., The boundary element method, volume 2: Applications in solids and structures, John Wiley and Sons, 2002.

Bonnet M., Equations intégrales et éléments de frontière en mécanique des solides, Ecole polytechnique, 1994.

Brebbia C.A., Dominguez J., Boundary elements: an introductory course, Mc Graw-Hill, $1^{\text {st }}$ edition, 1989.

Guiggiani M., "A general algorithm for the numerical solution of hypersingular boundary integral equations", ASME journal of applied mechanics, vol. 59, 1992, p. 604-614.

Kebir H., Roelandt J.M., Foulquier J., "A new singular boundary element for crack problems: Application to bolted joints”, Engineering Fracture Mechanics, vol. 62, 1999, p. 497-510.

Kebir H., Roelandt J. M., Gaudin J., «Simulation de la propagation de fissures dans les solides élastiques en modes mixtes par la méthode des équations intégrales duales », Revue européenne des éléments finis, vol. 9, nº 8, 2000.

Lachat J.C., A further developpement of the boundary integral technique for elastostatics, $\mathrm{PhD}$ thesis, University of Southampton, 1975.

Lachat J.C., Watson J.O., "Effective numerical treatment of boundary integral equations", International Journal for Numerical Methods in Engineering, vol. 10, 1976, p. 991-1005.

Leitao V., Aliabadi M.H., "The dual boundary element formulation for elastoplastic fracture mechanics”, International Journal for Numerical Methods in Engineering, vol. 38, 1995, p. 315-333.

Portela A., Aliabadi M.H., "The dual boundary element method: effective implementation for crack problems", International Journal for Numerical Methods in Engineering, 1992, p. 1269-1287. 
642 Revue européenne de mécanique numérique. Volume 16 - n 5/2007

Portela A., Aliabadi M.H., Rooke D.P., "Dual Boundary element analysis of cracked plates: singularity subtraction technique", International Journal of Fracture, vol. 55, 1992, p. 1728.

Standard test method for measurement of fracture toughness- ASTME 1820 (www.astm.org). 